\title{
Labyrinthe
}

28 | 2007 (3)

Des Juifs contre l'émancipation

\section{Refus des Lumières : Les penseurs du retour face au danger de la dissolution de l'identité juive}

Héloïse Hermant

\section{(2) OpenEdition}

12 Journals

Édition électronique

URL : http://journals.openedition.org/labyrinthe/2843

DOI : $10.4000 /$ labyrinthe.2843

ISSN : 1950-6031

Éditeur

Hermann

Édition imprimée

Date de publication : 21 décembre 2007

Pagination : 25-38

ISBN : 978-2-9526131-5-6

\section{Référence électronique}

Héloïse Hermant, «Refus des Lumières : Les penseurs du retour face au danger de la dissolution de l'identité juive », Labyrinthe [En ligne], 28 | 2007 (3), mis en ligne le 01 octobre 2009, consulté le 01 mai 2019. URL : http://journals.openedition.org/labyrinthe/2843 ; DOI : 10.4000/labyrinthe.2843

Propriété intellectuelle 


\title{
REFUS DES LUMIÈRES : \\ les penseurs du retour \\ face au danger de la dissolution de l'identité juive
}

\author{
Héloïse HERMANT \\ heloise.hermant@wanadoo.fr
}

Depuis deux décennies, un courant de pensée radical s'affirme autour de la «question juive». Animé paradoxalement (ou significativement) par de purs produits de la culture universitaire française, ce courant se mobilise face au constat d'une progressive dissolution de l'identité juive. Ces «penseurs du retour», fortement marqués par le magistère d'Emmanuel Levinas, aboutissent à une même conclusion foncièrement pessimiste: l'émancipation ne serait qu'un terme édulcoré visant à masquer un phénomène d'assimilation délétère pour l'identité juive ${ }^{1}$. La responsabilité en incomberait à la modernité née du long processus inauguré par la Révolution française et poursuivi par Napoléon Bonaparte, qui, en 1807, met en place le Grand Sanhédrin. C'est pourquoi les penseurs du retour font de 1789 et du «moment Napoléon» le point de départ d'une histoire retraçant l'influence pernicieuse des Lumières qui, sous des airs d'apparente neutralité, masquerait un christianisme diffus, imprégnant en profondeur tous les secteurs de la société.

1. Les penseurs du retour rejettent radicalement les Lumières - notamment en ce qu'elles seraient chrétiennes (pauliniennes) dans leur essence - et prônent un retour à la Torah. La dénonciation des Lumières et de la modernité ne constitue donc qu'un moment de leur pensée, dont le point de départ est la question de l'assimilation-dissolution. Dans ce sens strict, les figures d'Emmanuel Levinas et de Benny Lévy dominent. Il ne faut pas confondre ce courant avec celui qui récuse la modernité et construit les généalogies meurtrières d'une Europe antisémite, où les Lumières restent un référent positif opposé à l'obscurantisme. L'ouvrage de Georges Bensoussan, L'Europe. Une passion génocidaire. Essai d'histoire culturelle, Paris, Mille et une nuits, 2006, ne condamne pas les Lumières: celles-ci ne constituent significativement pas le terminus a quo de son raisonnement, puisqu'il cherche les sources du mal dès le Moyen Âge. Il déplore qu'elles n'aient pu enrayer la montée de la haine et de la violence et aient charrié un double négatif, les «anti-Lumières ». Quant à Jean-Claude Milner, s'il réfléchit d'abord en linguiste et n'accomplit pas le retour - la «teshuva»-, son radical rejet des Lumières rejoint les penseurs du retour stricto sensu. Sa pensée a largement irrigué celle de 
Les propos de Benny Lévy présentent les Juifs français comme inconsciemment contraints de se couler dans un moule identitaire forgé par le christianisme et les plaçant en porte-à-faux vis-à-vis d'eux-mêmes. Ils résonnent comme une condamnation sans appel de la modernité conçue comme un avatar du paulinisme:

Dans la notion de Juif français, il y a une espèce de bombe à retardement. [...] Un Juif français, tel qu'il s'est constitué depuis l'émancipation, depuis Napoléon, depuis le Consistoire, c'est quelqu'un qui estime qu'il est une particularité - juif - venue rejoindre l'universel - c'est-à-dire la culture et son idéal le plus haut, au moment décisif qu'a été l'après Révolution française, c'est-à-dire, au fond, croyait l'Israélite français, l'accomplissement du prophétisme d'Israël lui-même [...]. Ce modèle d'intégration-là, je disais que c'est une bombe à retardement. Pourquoi ? Parce que vous voyez bien que c'est faux [...]. Le Juif n'est pas une particularité qui rejoint l'universel $^{1}$.

\section{La légende dorée de l'émancipation a donc vécu. L'accroissement} des manifestations judéophobes, antisémites ou antisionistes, perceptible depuis le début des années $2000^{2}$, mis en série avec les pics de violence historique exercés contre les Juifs de France - dont l'affaire Dreyfus a valeur de symbole -, le tout relu à la lumière tragique de la

\footnotetext{
Benny Lévy, qui le cite à de nombreuses reprises, aussi bien dans Le meurtre du pasteur. Critique de la vision politique du monde, Paris, Grasset/Verdier, 2002, que dans Être juif. Étude lévinassienne, Lagrasse, Verdier, 2003. Milner donne à sa réflexion la forme d'une généalogie et noue la question de l'assimilation-dissolution à celle de l'extermination. Enfin, une dernière strate de penseurs interagissent avec les chantres du retour: ils pointent les ambivalences de la modernité et la part d'ombre des Lumières, sans toutefois s'y amalgamer. Ces sociologues (le Shmuel Trigano de L'Avenir des Juifs de France, Paris, Grasset, 2006 et la revue Controverses), linguistes (Henri Meschonnic dans L'Utopie du Juif, Paris, Desclée de Brouwer, 2001) ou historiens (Monique-Lise Cohen, Les Juifs ont-ils du cæur? Discours révolutionnaire et antisémitisme, Toulouse, Vent Terral, 1992), s'ils raisonnent à partir d'une trame événementielle, ne bâtissent pas de systèmes comme Milner. Ils préfèrent souligner les ambiguïtés du projet révolutionnaire des Lumières, ou s'attaquer à des manifestations de l'antisémitisme contemporain et d'un certain malaise régnant au sein de la communauté juive de France, qui s'expliquent en grande partie par l'évolution sociologique - jamais posée comme donnée d'avance ou irréversible - de la France née des Lumières avec la laïcité et la proclamation des droits de l'homme.

1. Alain Finkielkraut et Benny Lévy, Le Livre et les livres. Entretiens sur la laïcité, Lagrasse, Verdier, 2006, p. 84.

2. L'interprétation de ces événements dans leur globalité (incendies contre des synagogues, agressions antisémites, cimetières profanés...), informée par le contexte international (Intifada Al-Aqsa, conférence de Durban, 11 septembre 2001...), fait l'objet de débats et de polémiques sur l'importance et la nature du phénomène.
} 
Shoah, convainc les penseurs du retour soit de révéler des blocages structurels nés des Lumières, soit de saisir dans un vaste continuum tous ces épisodes ${ }^{1}$. Dans ce dernier cas, ils cherchent à déceler la logique cachée qui présiderait à leur avènement et lierait irrémédiablement ces événements entre eux. Certains élaborent ainsi des herméneutiques totalisantes. Le Jean-Claude Milner des Penchants criminels de l'Europe démocratique met aux prises une Europe hypostasiée cherchant à apporter par tous les moyens une «solution» au «problème juif» : celui-ci prend dans sa forme la plus brutale, scandaleuse et inhumaine, le visage de l'extermination nazie et, dans sa forme la plus insidieuse, celui de l'émancipation porteuse d'assimilation. Intégration, assimilation, dissolution, telles seraient les étapes que les Juifs se verraient condamnés à suivre pour être sacrifiés sur l'autel de l'Universel. Milner ne montre pas autre chose que les métamorphoses de la «machine à assimiler» de l'émancipation, lorsqu'il décrit les figures successives du «Juif de savoir», du «Juif des droits de l'homme» puis du «Juif de négation $»^{2}$.

L'épanouissement de ce courant de pensée du retour en cette fin du $\mathrm{XX}^{\mathrm{e}}$ siècle français n'est pas fortuit. Si le projet même des promoteurs de l'émancipation renfermait des ambiguittés dérangeantes et des implicites incompatibles avec l'identité juive - le texte de l'Abbé Grégoire en offre une illustration flagrante -, il a fallu qu'un certain nombre d'événements jalonnent le $\mathrm{XX}^{\mathrm{e}}$ siècle pour qu'une poignée d'auteurs prenne conscience des pièges tendus par les Lumières. Emmanuel Levinas fut l'un des premiers à parler du processus de «déjudaïsation» que l'émancipation impliquait. Évoquant l'accession à la citoyenneté des Juifs français et leur pleine adhésion à la philosophie de l'émancipation, vécue comme un «acte métaphysique», un «acte solennel retentissant sur leur vie intérieure», il écrit:

C'est à partir de cette essence exceptionnelle de la France où vie politique et vie morale se rejoignaient, à partir des idéaux de la Révolution de 1789

\footnotetext{
1. Pour Jean-Claude Milner, la conférence de Durban (31 août-8 septembre 2001) et les défilés contre la guerre d'Irak en France et en Europe au début de 2003, où la dénonciation d'Israël a été un thème récurrent, constituent le terminus ad quem de la généalogie «criminelle» contre le «nom "Juif"» de l'Europe démocratique, qu'il établit. Voir Jean-Claude Milner, Les Penchants criminels de l'Europe démocratique, Lagrasse, Verdier, 2003, p. 94-101.

2. Jean-Claude Milner, Le Juif de savoir, Paris, Grasset, 2006.
} 
et de la Déclaration des droits de l'homme, à travers la littérature et les institutions qui l'exprimaient, que l'attachement s'étendait à l'histoire et aux paysages générateurs de ces idées, jusqu'à devenir la conscience d'un enracinement végétal où bien des israélites français auront oublié la source religieuse de leur amour, désormais enfants du terroir, autochtones, Français aussi naturellement français que les prés sont verts et les arbres en fleurs ${ }^{1}$.

Levinas prend la plume peu après la guerre des Six-Jours, afin de méditer sur les répercussions de cet événement sur la situation des Juifs de France. La sympathie de ces derniers pour l'État hébreu et leur désaccord avec le gouvernement français sur ce point de la politique internationale leur ont valu d'être accusés de duplicité et de «double allégeance». Pour certains, les Juifs s'excluaient eux-mêmes de la communauté nationale, en signifiant leur attachement à Israël. Le discours de De Gaulle en novembre 1967, qui qualifie les Juifs de «peuple sûr de lui et dominateur», est ressenti comme un divorce entre les Juifs et la nation française. Dans ce contexte chargé, Levinas livre un regard rétrospectif de leurs rapports sous le régime de l'émancipation. Ce faisant, il restitue les événements qui l'ont conduit à dresser le constat de la dissolution de l'identité juive et à formuler une série de questions capitales qui sous-tendent le thème de l'émancipation: "Qu'est ce qu'être juif?», «Peut-on être un Juif émancipé ?» et enfin «Existe-t-il une nouvelle façon d'être juif dans la France de 1967 ?». C'est qu'il est des événements qui «brûlent les concepts qui expriment leur substance $^{2} »$, qui rendent visibles les contradictions de la configuration née de l'émancipation en exacerbant certaines tensions jusque-là cachées: l'extermination nazie et la Shoah, la création d'Israël et la question de la laïcité avec ses aléas en France. Chacun d'eux oblige à repenser l'identité juive et sa compatibilité avec le modèle français de l'émancipation, et, au-delà, avec la «modernité». La Shoah suscite, notamment, une réflexion sur l'universalité du peuple juif dans son rapport au mal. Paradoxalement, elle a pu ainsi permettre l'érection d'un théâtre de la mort de Dieu, responsable d'un quasi renoncement à la Torah par l'affirmation de la souffrance (attitude que condamne Benny Lévy). La création de l'État d'Israël, elle, réinstalle l'identité juive dans une his-

1. Emmanuel Levinas, «L'espace n'est pas à une dimension», dans Difficile Liberté. Essais sur le judaïsme, Paris, A. Michel, 1963, rééd. Paris, Librairie générale française, 1984, p. 388.

2. Ibidem, p. 390. 
toricité forte en reposant la question du messianisme et la question de l'hébreu. Par ailleurs, elle fissure le modèle du Juif universel et incite à s'interroger sur les liens entre peuple, nation et État. Enfin, les difficultés auxquelles se trouve confronté le modèle laïc français soulignent l'écart qui sépare «confession» et «religion», conviction du for intérieur et injonction extérieure, «foi» et «étude».

Il s'agit donc, pour nous, de restituer «en mouvement» ces pensées du retour en France, à la fin du Xx ${ }^{e}$ et au début du XXI ${ }^{\mathrm{e}}$ siècle. Afin de ne pas tomber dans le catalogue, nous ne partirons pas de la réflexion d'un penseur en particulier, mais nous examinerons des couples en tensions nés des «pièges» des Lumières, chacun d'entre eux pouvant enclencher un dispositif de dissolution de l'identité juive. Nous étudierons successivement la question de l'universel, clef de voûte des Lumières, puis l'antagonisme entre religion et confession saisie à travers la question de la laïcité 1 . Le problème du rationalisme et des rapports entre religion et philosophie, thème transversal, constituera la ligne de fond de ces débats. Ces pierres d'achoppement permettront de ne jamais perdre de vue la question de l'émancipation (où d'aucuns voient un prélude à l'assimilation et à la dissolution), tout en introduisant dans les débats les positions des tenants de l'émancipation ou des penseurs hostiles à l'idée d'une singularité juive.

\section{L'universel en question : l'implacable logique d'assimilation des Lumières}

La tragédie de la Shoah et la création de l'État d'Israël ont eu des effets contradictoires sur l'image que l'Occident a façonnée du peuple juif. Alors que la mémoire de l'extermination nazie avait pu ériger le Juif en incarnation de l'humanité souffrante en proie au mal, en symbole de l'universel'2, la création d'Israël, en tant qu'État caractérisé par un particularisme ethnico-religieux, a fissuré, dans les esprits, cette

1. Il faut prendre le couple confession-religion comme une interface, et non comme une opposition stricte. Le judaïsme n'est pas qu'une religion, mais l'assimilation à laquelle incitent les Lumières entre religion et confession prive implicitement le judaïsme de ses autres dimensions (politique, sociale, ...) et le dénature ainsi par ce travail de phagocytage.

2. Il n'est que d'écouter Levinas, «De la montée du nihilisme au juif charnel», ibidem, p. 331 : «Après vingt siècles d'existence anachronique, le judaïsme redevenait le théâtre de la Divine Comédie.» 
figure de l'universel. Une lecture répandue du conflit israélo-palestinien a porté à incandescence cette tension, ravivant le débat autour de cette question de l'universel. Certains penseurs du retour ont ainsi été incités à expliciter par ce prisme leur refus des Lumières et du processus d'assimilation qu'elles portaient dans leurs flancs ${ }^{1}$.

La violence des discours qui scandent la «querelle du nom "Juif" » s'explique par l'ampleur des enjeux. Au delà du «paulinisme ${ }^{2}$ », c'est l'Occident comme concrétisation et produit des Lumières qui se trouve sur la sellette. La polémique creuse l'arène où s'affrontent deux régimes de pensée inconciliables, deux façons d'appréhender le lien du soi au tous décliné dans sa dimension logique, politique et théologique.

Pour Alain Badiou, la Shoah aurait consacré le mot «juif» en un signifiant destinal, le plaçant hors de tout maniement ordinaire des prédicats d'identité ${ }^{3}$ et établissant une transcendance communautaire incompatible avec l'universalisme contemporain ${ }^{4}$. Forts d'une idéologie victimaire présentant Hitler comme le mal absolu, les Juifs bénéficieraient de la sacralisation de leur nom, afin d'imposer une transcendance fictive par l'État hébreu qui, «dans sa prétention identitaire fermée à être un État juif et à tirer de cette prétention des privilèges » au détriment des Palestiniens ${ }^{5}$, refuserait d'entrer dans l'universel par l'ostentation d'une irréductible singularité6. La conclusion donne l'estocade: en affichant un prédicat religieux, «l'État d'Israël est la forme extérieure, de nature coloniale, qu'a prise la sacralisation du nom des juifs ${ }^{7} »$.

Biffer le nom «Juif» au nom d'une «fausse immédiateté de l'universel $^{8} \gg$ fait, pour Jean-Claude Milner, apparaître les «penchants criminels de l'Europe démocratique», et correspond chez Badiou, à une sécularisation du paulinisme ${ }^{9}$. Milner vitupère contre les Lumières,

\footnotetext{
1. Nous ne traitons pas ici du conflit israélo-palestinien pour lui-même, mais l'appréhendons qu'en tant que symptôme de conceptions divergentes de l'universel, amenant des penseurs du retour à remettre en cause certaines valeurs des Lumières.

2. Selon Paul: «Il n'y a plus ni juif ni grec, il n'y a plus ni esclave, ni libre, il n'y a plus ni homme ni femme, car vous êtes un en Jésus-Christ» (Gal. 3, 28). Traversant les particularismes, Paul érige l'universel sur des «chaque-un» qui deviennent «un».

3. Alain Badiou, Portées du mot «juif», Circonstances, 3, Paris, Lignes et Manifeste, 2005, p. 9.

4. Ibidem, p. 10 et p. 58.

5. Ibidem., p.15.

6. Ibidem, p. 11.

7. Ibidem, p. 25.

8. Jean-Claude Milner, Les Penchants criminels de l'Europe démocratique, op. cit., p. 108.

9. Ibidem, p. 103.
} 
chrétiennes dans leur essence dans la mesure où elles s'inscrivent dans le prolongement de l'apôtre. Coupable d'avoir institué le Juif en problème structural appelant une question et générant une violence inouïe contre le nom « Juif ${ }^{1} »$, l'idéologie des Lumières exacerberait la dynamique d'assimilation dans la mesure où tout démenti à la conception de l'universel qu'elle a bâtie la minerait de l'intérieur. C'est bien le point dont débat Milner, qui analyse cet «universel facile» permettant de dissoudre l'un dans le tout en niant les singularités, et procédant par englobements successifs parce qu'il se dit illimité et, partant, parce qu'il ne tolère aucune limite. Le linguiste affirme que la vulgate aristotélicienne forge un paradigme logico-politique européen, puisant à deux sources. La première, l'Organon, réfléchit sur le tout comme règle de la pensée, sous la forme de l'universel. La seconde, la Politique, pense le tout comme règle des divers rassemblements humains. La doctrine de Paul permettrait de cimenter le système, en ce qu'elle réaliserait l'amalgame - étranger à la pensée du Stagirite - du tous logique et du tous politique, pour imbriquer langue logique, langue théologique et langue politique. Mais l'assimilation du tous politique et du tous logique transmuant le quelconque dans la figure plus noble de l'universel se trouverait ébranlée par la survivance du Juif dans son irrémédiable singularité. C'est dans cette perspective que Milner interprète l'hostilité que suscite en Occident l'État hébreu².

Face au tout illimité des Lumières, qui traque partout le même et se veut en perpétuelle expansion, il existerait un tout limité, celui qu'incarne le nom «Juif», qui articule autrement le singulier et l'universel. Résistant contre la dynamique de dissolution du nom «Juif» dans un grand tout de l'indéterminé issue de l'émancipation, certains penseurs du retour édifient le particularisme comme universel. La réponse la plus complète formulée à l'encontre des Lumières est probablement celle d'Emmanuel Levinas. Ce dernier pense une «universalité de rayonnement» et non plus d'englobement, sise sur la particularité d'Israël définie comme l'étude de la Torah ${ }^{3}$. Levinas distingue, d'une part, le geste

1. Ibidem, p. 9.

2. Ibidem, p. 97-98.

3. Le rapport de la singularité juive à l'universel n'a rien d'une extrapolation complaisante. Pour Levinas, la question de l'universel est consubstantielle au judaïsme: «C'est là notre universalisme. Dans la caverne où reposent les Patriarches et nos mères, le Talmud fait reposer Adam et Ève: c'est pour l'humanité toute entière que le judaïsme est venu.» Emmanuel Levinas, «Israël et l'universalisme», op. cit., p. 266. 
originel de la pensée occidentale, christique puisqu'il correspond à la destruction de la transcendance et à l'exaltation de la figure du Fils comme auto-engendrement, et, d'autre part, le geste fondateur du judaïsme d'accueil de la Révélation sur le Sinaï, par l'obéissance au commandement et soumission au joug de la loi à travers la figure du Père ${ }^{1}$. Le judaïsme se définit précisément par ce geste incompréhensible du reste de l'humanité au point de la scinder en deux, qui consiste à obéir avant de comprendre et qui dresse cette hétéronomie en pierre de touche de la liberté et de l'universalité. Dans une logique antinomique, l'Occident et les Lumières mettent en tension raison et révélation. Ils estiment que seule l'autonomie de l'esprit, seules la pensée auto-engendrée et la raison ne puisant qu'en elle-même, en un mot, seul le discours du Fils est non aliénant et universel car désenclavé de tout particularisme. On voit mieux pourquoi, selon Levinas, les Juifs ne peuvent accepter l'universel des Lumières, sous peine de se renier. Tout l'effort du philosophe se résumera dès lors à bâtir une éthique fondée sur la singularité incarnée par le visage d'autrui, à la fois vulnérable et inviolable ${ }^{2}$. Le philosophe rejette la démarche occidentale de conceptualisation qui ne saisit des choses que ce qu'elles ont de général et réduit l'autre au même. À rebours de Hegel et de sa dialectique assimilée à une forme de parousie chrétienne, Levinas fond la singularité dans un universel - le visage, c'est l'humanité - dépeint comme un infini qui outrepasse la totalité et ouvre sur l'au-delà ${ }^{3}$ : «La justice rendue à l'autre, me donne de Dieu une proximité indépassable ${ }^{4}$.»

\section{Les pièges politiques des Lumières : l'émancipation, les droits et la Loi}

Pour les penseurs du retour, l'analyse de l'universel comme principe moteur des Lumières rend donc manifeste l'inextricable association du

\footnotetext{
1. Emmanuel Levinas, «Être juif», Cahiers d'études lévinassiennes, 1, p. 102.

2. Emmanuel Levinas, Totalité et infini. Essai sur l'extériorité, Paris, 1990.

3. Et non pas d'une totalité infinie, expression de l'au-delà. Sur la façon dont Levinas traite le legs philosophique de Hegel, on pourra consulter Emmanuel Levinas, «Hegel et les juifs », Difficile Liberté, op. cit., p. 352-357. Pour mieux comprendre l'influence de Rosenzweig sur Levinas, à travers le mouvement de déprise de la dialectique hégélienne qu'opère le philosophe allemand, voir Emmanuel Lévinas, «Entre deux mondes», ibidem, p. 272-302.

4. Emmanuel Levinas, «Une religion d'adultes», ibidem, p. 38.
} 
logique, du politique et du religieux. Si les Lumières, en surgeon du paulinisme, engendrent une pensée totalitaire et «parricide» (dans un sens christologique), incompatible avec la singularité juive, comment recevoir les Lumières dans leur émanation politique: l'État-nation, les droits de l'homme et le suffrage universel?

L'héritage de la Révolution française ne paraissait pourtant pas menacer l'identité juive de dissolution. Grâce à l'émancipation, qui fait des Juifs des citoyens à part entière, grâce à la séparation du politique et du religieux, une grande entente devenait possible entre les hommes en dehors de la religion désormais cantonnée au privé. S'entrebâillait la porte d'une ère de fraternité entre citoyens libres réunis non plus par leur attachement à un sol, mais autour de valeurs et d'idées. Une telle conception adulte et irénique du lien social résonnait en parfaite harmonie avec le judaïsme tel que le définissait Levinas: une religion de responsabilité mettant en synonymie lien religieux et lien éthique et tendant à bâtir une société juste où cohabiteraient la grande fraternité des hommes et au sein de l'État-nation, des citoyens. Néanmoins, le bilan de l'émancipation affiche des résultats alarmants: désertion des synagogues, pauvreté de la pensée juive contemporaine, prédominance d'un judaïsme conçu non plus comme une religion, ni même une culture, mais comme l'accumulation inerte de «souvenirs de famille ${ }^{1}$ », voire comme une sensibilité diffuse animée parfois d'un élan de solidarité pour les coreligionnaires persécutés. Pour les penseurs du retour, le processus de dissolution de l'identité juive passe par le triomphe de ce judaïsme-reliquat en sursis.

Quels sont les ressorts cachés de cette dynamique mortifère? Dans leur majorité, les penseurs du retour n'en cherchent pas la clef dans une analyse des discours révolutionnaires. Si l'on excepte Milner, qui, lui, se place à l'échelle européenne, ils ne traquent pas non plus de principes - de «penchants»- occultes dont ils déchiffreraient l'accomplissement en déroulant une trame factuelle. Les événements qui rappellent les Juifs français à leur singularité et leur donnent l'occasion de se «ressaisir», sur un mode conflictuel voire tragique, ont autant pour cause la bêtise, l'intolérance, la barbarie qui menacent sans cesse de sourdre selon une dynamique pulsatile, et non sérielle, que des incompatibilités structurelles, issues des Lumières et délétères, non pas parce

1. Emmanuel Levinas, «L'assimilation aujourd'hui », ibidem, p. 383. 
qu'elles exprimeraient la mise en œuvre d'un dessein caché de destruction, mais parce qu'elles emprisonneraient les Juifs dans un modèle aliénant, à leur insu. En France, le régime politique mis en place par les Lumières ne serait pas criminel au sens d'assassin, mais pousserait au suicide par aliénation. La question est donc celle de l'assimilation.

Voyons plus précisément ce qu'il en est. Les Lumières promeuvent l'abolition des singularités dans le tout illimité de la société présentée comme l'universel, ainsi que la non hétéronomie de la pensée. La démocratie du suffrage universel réalise le premier point de ce programme; la séparation du politique et du religieux, le second. Les droits de l'homme, eux, subsument les deux pans du diptyque. Ce faisant, l'émancipation signe la victoire des droits sur la loi et provoque une série de conséquences menaçant l'identité juive.

Ainsi, Benny Lévy dénonce dans la séparation du politique et du religieux un geste christologique. Le «meurtre du pasteur ${ }^{1} »$ transforme la parole des Anciens fondée sur l'autorité de la sagesse en parole politique qui cherche à séduire parce qu'elle n'aura de consistance qu'en emportant l'assentiment du plus grand nombre. La rationalité des Lumières implique donc la laïcisation et prolonge le destin politique du logos. Or, selon Lévy, la chance et la singularité des Juifs sont d'avoir échappé à ce régime de parole. L'affrontement d'Alexandre le Grand et de Shimon le Juste signale que la parole juive est restée nouée à la sagesse des maîtres. Quant à l'adage du Talmud, «le maître est plus qu'un prophète », il signifie que le maître accède au contenu de la prophétie par le biais de la sagesse, non pas par la réception de l'En-haut comme le prophète, mais par un «travail qui, du bas, nous arrache à l'obscurité et retrouve l'éclat de la parole de $\mathrm{feu}^{2} »$. La soumission au joug de la loi, l'observance stricte du rite ne dissocient pas le religieux du politique qui se fonde dans la parole du sage ${ }^{3}$. La laïcité rend le Juif étranger à lui-même parce qu'elle le conduit à son insu à parler un langage qui trahit le sien.

Le rejet de l'hétéronomie et le triomphe des droits sur la loi causent donc pour les Juifs une non coïncidence de soi à soi mortelle à terme, parce qu'elle transforme la religion en confession cantonnée à la sphère

1. Benny Lévy, Le Meurtre du pasteur, op. cit.

2. Alain Finkielkraut et Benny Lévy, op. cit., p. 40.

3. Levinas ne dit pas autre chose lorsqu'il assimile lien politique et lien éthique dans une religion de justice. 


\section{Refus des Lumières}

du privé et à laquelle on adhère parce qu'on y croit. Levinas décrit bien ce phénomène d'assimilation porteur de dissolution:

L'esprit occidental auquel le Juif s'est assimilé pour ne plus toucher que la surface du judaïsme se définit, peut-être, par le refus de toute adhérence sans acte d'adhésion. [...]. Tout attachement exceptionnel est travaillé pour lui par le soupçon d'être partagé par tous. Il faut dès lors ne pas s'accepter spontanément et, par conséquent, commencer par prendre distance à l'égard de soi, se regarder du dehors, réfléchir sur soi; se comparer aux autres, réduire donc cette identité personnelle qu'on est en autant d'indices, d'attributs, de contenus, de qualités, de valeurs; s'analyser, se monnayer ${ }^{1}$.

Ailleurs, il rappelle l'absurdité d'un judaïsme ramené à un credo: «une vraie culture ne peut se résumer, car elle réside dans l'effort même qui la cultive ${ }^{2} »$. Quoi de commun entre cette confession fossile et un judaïsme vécu comme une injonction, un commandement, et qui se réalise dans le quotidien de l'étude et de l'observance de la loi ?

Il faut donc admettre que la société française, derrière des apparences de neutralité, conserve dans ses formes sécularisées une atmosphère chrétienne qui agit par imprégnation ${ }^{3}$. Selon Levinas, un des effets les plus graves consiste certainement en une «domestication» du Divin. La séparation du religieux et du politique se traduit par un spirituel aseptisé, un compartimentage étanche entre les activités quotidiennes et une vie intérieure déliée de toute responsabilité. C'est, pour Levinas, oublier que c'est parmi les hommes, face au visage d'autrui, que le Juif prend conscience de l'au-delà. Le Juif ne se situe jamais hors du monde 4 .

Enfin, l'émancipation offre au Juif une liberté de choix radicale et séduisante, où le refus d'une pensée sans adhésion se traduit nécessairement par un passage par l'athéisme (je feins que je ne crois pas avant de décider si je crois), qui ne dispense aucun garde-fou contre le nihilisme. En favorisant une sortie du religieux, le régime politique instauré par les Lumières nourrit chez les citoyens matérialisme et individualisme.

1. Emmanuel Levinas, «Pièces d'identité», Difficile Liberté, op. cit., p. 87.

2. Emmanuel Levinas, «Comment le judaïsme est-il possible?», ibidem, p. 376.

3. Emmanuel Levinas, ibidem, p. 367.

4. Ibidem, p. 370. 


\section{Difficile Liberté}

Au final, pour les penseurs du retour, le mal du Juif émancipé vient de ce qu'il emboîte la totalité de son existence dans les catégories occidentales de «nation», «État», «confession», «profession», «classe sociale»..., autant de catégories mal taillées pour lui, qui le confondent. Et l'on pointe là des questions épineuses : qu'est-ce qu'être juif? les Juifs sont-ils un peuple, une nation, une communauté, une religion, une culture reliquat? peut-on être juif et français? peut-on être un Juif émancipé? Car c'est lorsque le Juif se dit et se vit citoyen, lorsqu'il vibre à l'unisson de la mystique nationale, lorsqu'il pense avoir prise sur son temps, que les contradictions s'exacerbent.

Un tel déchirement de l'âme juive est-il irrémédiable? Les réponses divergent. Pour Benny Lévy qui incarne ici une des lignes les plus fermes de la pensée du retour, on ne peut être juif et français ${ }^{1}$. La seule réalité du Juif est la connaissance de la Torah, c'est donc en étranger que ce dernier obéit aux lois de l'État français². Le Juif n'est pas une question appelant une solution parce qu'il entrave l'extension de l'universel, comme le lui suggère l'Europe des Lumières. Il est dans sa facticité, et ce depuis que 600000 Hébreux ont découvert ensemble, sur le Sinaï, l'unicité de chacun. Le Juif doit se consacrer entier à accomplir les mitzvot s'il veut rester juif dans un mouvement de repli du monde seul capable de lui rendre son indépendance ${ }^{3}$.

1. Benny Lévy reproche à Levinas d'avoir opéré la conversion philosophique du judaïsme en ayant
dilué la révélation dans «l'humanisme de l'autre homme». Il interprète l'ouverture vers l'au-delà que
permet la confrontation avec le visage d'autrui comme une coïncidence intolérable entre mort et infini.
En faisant de la Shoah une religion qui oppose le Juif angélique mort à l'imperfection du Juif vivant,
Levinas ne présente pas l'au-delà comme une positivité, une présence pleine; il prive le Juif de toute
consolation. En somme, Levinas continuerait à philosopher, il aurait simplement reversé l'étymolo-
gie de la philosophie qui ne serait non plus amour de la sagesse mais sagesse de l'amour. Ces points
font l'objet de Visage continu. La pensée du retour chez Emmanuel Levinas, Lagrasse, Verdier, 1998,
et de l'ouvrage Être juif, op. cit.
2. Le fait, pour un Juif, d'être un citoyen français et de vivre en France n'affecte nullement son essen-
ce, qui se résume à sa judéité. C'est en ce sens seulement qu'on ne peut être juif et français. Cela ne
signifie pas qu'un Juif ne peut vivre en France. Au contraire, selon Lévy, le régime né de l'émanci-
pation offre un cadre favorable à l'étude de la Torah. Le tout est d'être conscient des pièges des
Lumières et de ne pas laisser dissoudre l'identité juive sous peine de devenir ce que Lévy nomme un
«Juif imaginaire», reprenant le titre du fameux livre d'Alain Finkielkraut, Le Juif imaginaire, Paris,
Le Seuil, 1980 .
3. Lévy se montre très véhément sur cet aspect. Voir Alain Finkielkraut et Benny Lévy, op. cit., p. 88. 
Aux antipodes, Alain Finkielkraut juge la chose possible ${ }^{1}$. Car pour lui, si une dialectique du limité et de l'illimité est bien à l'œuvre, il ne s'agit pas d'un dispositif pervers intrinsèquement lié aux Lumières, mais d'un dévoiement conjoncturel du droit transmué en «les droits », coupable d'une opposition fallacieuse entre bonne autonomie et mauvaise hétéronomie. La laïcité ne se réduit pas à la séparation du religieux et du politique. Elle est ce qui permet l'indépendance totale du spirituel: «La liberté de l'esprit n'est pas la liberté d'opinion mais la possibilité pour l'esprit d'atteindre la vérité sans autorité extérieure ${ }^{2}$.» Cependant, le fractionnement de la nation en une mosaïque de communautés exacerbant leurs particularismes dresserait contre le droit exigeant l'obéissance aux limites qu'il pose, les droits servant la soif d'expansion des individus. Le pluralisme ne correspondrait plus à la cohorte des voies en quête de vérité, mais à «l'exhibition péremptoire des identités» où la «reconnaissance se substitue à la connaissance ${ }^{3}$ ». Quant à Levinas, il choisit la voie médiane, en partant de l'idée que l'élection du peuple juif consiste dans l'articulation unique qu'il réalise du singulier et de l'universel:

La communauté juive est, par contre, une communauté qui a l'éternité dans sa nature même. Elle ne tient son être ni d'une terre, ni d'une langue, ni d'une législation soumise aux renouvellements et aux révolutions. Sa terre est «sainte» et terme d'une nostalgie, sa langue est sacrée et n'est pas parlée. Sa Loi est sainte et n'est pas une législation temporaire, faite pour la maîtrise politique du temps. Mais le juif naît juif et est confiant en la vie éternelle dont il vit la certitude à travers les liens charnels qui le rattachent à ses ancêtres et à ses descendants ${ }^{4}$.

Pour Lévinas, une communauté, un peuple sans État, diasporique, échappant à la contingence de l'histoire parce qu'il vit sa loi invariable

1. Alain Finkielkraut émet des réserves face à l'injonction de Benny Lévy qui tendrait à réduire l'action des Juifs à rester juifs coûte que coûte et à avoir ainsi une vocation tautologique. Contre Lévy qui prône la séparation, Finkielkraut suit Levinas sur l'idée d'un judaïsme participant au monde pour favoriser son humanisation. Voir «La déplorable affaire du foulard», dans Alain Finkielkraut et Benny Lévy, ibidem.

2. Alain Finkielkraut et Benny Lévy, ibidem, p. 65-66.

3. Ibidem, p. 92-93.

4. Emmanuel Levinas, «Entre deux mondes», Difficile Liberté, op. cit., p. 291. Voir aussi p. 298. 
- où la séparation du politique et du religieux n'a pas grand sens - dans une vie rituelle cyclique qui recouvre une importance ontologique ${ }^{1}$. Un peuple du Livre et non de la terre où la liberté à l'égard des formes sédentaires de l'existence relègue au loin les valeurs de l'enracinement. Voilà la façon humaine dont les Juifs sont au monde en instaurant les formes de responsabilités les plus hautes ${ }^{2}$. Alors, pour Levinas, on peut être un Juif émancipé si l'on sait maintenir l'équilibre fragile entre engagement et désengagement, si l'on assume qu'on est «une non-coïncidence avec son temps, dans la coïncidence: au sens radical du terme, un anachronisme, la simultanéité d'une jeunesse attentive au réel et impatiente de le changer et d'une vieillesse ayant tout vu, remontant à l'origine des choses ${ }^{3}{ }^{3}$, si l'on peut retrouver dans le souffle et le rythme des lettres carrées, la sagesse et le savoir où toutes les générations se confondent. En deux mots, une «difficile liberté»! Car c'est là que tout commence. Pour nos auteurs, on ne saurait se pétrifier dans le mouvement même du retour qui tient plutôt de la volte-face. La dénonciation des Lumières occidentales n'est qu'un préliminaire, souvent exprimé sur un mode polémique, qui ne vaut que par la prise de conscience qu'elle doit provoquer. Ce sont davantage les sociologues, les historiens ou les linguistes qui s'y étendent. Pour ceux qui accomplissent le retour, il ne s'agit pas en soi de faire de la dénonciation des Lumières et des effets pervers de l'émancipation la matrice d'une philosophie de la Révélation. Pour eux, seul importe finalement de penser autrement et de retrouver la Torah pour qu'elle redevienne la sève de la vie.

1. On voit quels problèmes soulève la création de l'État d'Israël. Après des siècles d'innocence politique, les Juifs verraient-ils dans la forme de l'État-nation occidental celle de leur accomplissement? Tragique ironie, diront les uns. À moins d'y voir une possibilité de s'inscrire dans l'histoire pour réaliser un monde juste? Levinas consacre une réflexion à l'État d'Israël dans À l'heure des nations, Paris, Éditions de Minuit, 1988.

2. Voir Emmanuel Levinas, «Une religion d'adultes», Difficile Liberté, op. cit.

3. Emmanuel Levinas, «Judaïsme et temps présent», ibidem, p. 318. 